TRANSACTIONS OF THE

AMERICAN MATHEMATICAL SOCIETY

Volume 366, Number 3, March 2014, Pages 1213-1235

S 0002-9947(2013)05843-6

Article electronically published on August 2, 2013

\title{
CONTINUOUS AND DISCRETE FOURIER FRAMES FOR FRACTAL MEASURES
}

\author{
DORIN ERVIN DUTKAY, DEGUANG HAN, AND ERIC WEBER
}

\begin{abstract}
Motivated by the existence problem of Fourier frames on fractal measures, we introduce Bessel and frame measures for a given finite measure on $\mathbb{R}^{d}$, as extensions of the notions of Bessel and frame spectra that correspond to bases of exponential functions. Not every finite compactly supported Borel measure admits frame measures. We present a general way of constructing Bessel/frame measures for a given measure. The idea is that if a convolution of two measures admits a Bessel measure, then one can use the Fourier transform of one of the measures in the convolution as a weight for the Bessel measure to obtain a Bessel measure for the other measure in the convolution. The same is true for frame measures, but with certain restrictions. We investigate some general properties of frame measures and their Beurling dimensions. In particular, we show that the Beurling dimension is invariant under convolution (with a probability measure) and under a certain type of discretization. Moreover, if a measure admits a frame measure, then it admits an atomic one, and hence a weighted Fourier frame. We also construct some examples of frame measures for self-similar measures.
\end{abstract}

\section{Contents}

1. Introduction

2. Qualitative structure results

3. Beurling dimension

4. Affine iterated function systems

References

\section{INTRODUCTION}

A fundamental result of Fourier analysis is the Plancherel theorem: for Lebesgue measure $\lambda$ on $\mathbb{R}$,

$$
\int_{\mathbb{R}}|\hat{f}(t)|^{2} d \lambda(t)=\int_{\mathbb{R}}|f(x)|^{2} d \lambda(x) .
$$

This equality suggests the idea of $\lambda$ as a dual measure to itself, in that the norm of the Fourier transform of $f$ is equal to the norm of $f$. Similarly, for the measure $m=\left.\lambda\right|_{[0,1]}, \lambda$ is a dual measure in the same sense, since if $f$ is supported on $[0,1]$,

Received by the editors November 9, 2011.

2010 Mathematics Subject Classification. Primary 28A80, 28A78, 42 B05.

Key words and phrases. Plancherel theorem, frame, Bessel, Fourier series, Hilbert space, fractal, self-similar, iterated function system.

This research was supported in part by the National Science Foundation grant 1106934. 
then equation (1.1) holds. Yet there is another measure that satisfies this norm equivalence, namely the measure $\nu=\sum_{n \in \mathbb{Z}} \delta_{n}$, since

$$
\int_{\mathbb{R}}|\hat{f}(t)|^{2} d \nu(t)=\sum_{n \in \mathbb{Z}}\left|\int_{\mathbb{R}} f(x) e^{-2 \pi i n x} d m(x)\right|^{2},
$$

which equals $\int_{\mathbb{R}}|f(x)|^{2} d m(x)$ by virtue that the integer exponentials form an orthonormal basis for $L^{2}[0,1]$. Moreover, for any sequence $\left\{e^{2 \pi i \gamma_{n} x}: n \in \mathbb{Z}\right\}$ which forms a Fourier frame [DS52, OCS02] for the Paley-Weiner space, $\nu=\sum_{n \in \mathbb{Z}} \delta_{\gamma_{n}}$ will be a dual measure in the slightly more general sense that $\|f\|_{L^{2}(m)} \simeq\|\hat{f}\|_{L^{2}(\nu)}$. In this paper, we consider questions of existence and structure of dual measures for singular measures, in particular measures which are invariant under iterated function systems.

In general, we consider a Borel measure $\nu$ on $\mathbb{R}^{d}$ to be dual to $\mu$ if for every $f \in L^{2}(\mu)$,

$$
\int_{\mathbb{R}^{d}}|\widehat{f d \mu}(x)|^{2} d \nu(x) \simeq \int_{\mathbb{R}^{d}}|f(t)|^{2} d \mu(t)
$$

where the Fourier transform is given by

$$
\widehat{f d \mu}(t)=\int f(t) e^{-2 \pi i t \cdot x} d \mu(x) \quad\left(t \in \mathbb{R}^{d}\right)
$$

for a function $f \in L^{1}(\mu)$. As we shall see, this is a generalization of the idea of a Fourier frame.

This idea of dual measures is closely related to other concepts. Jorgensen and Pedersen consider spectral pairs in [JP99. These spectral pairs consist of two measures $\mu$ and $\nu$ on $\mathbb{R}^{d}$ such that the equivalence in equation (1.2) is an equality together with the requirement that the Fourier transform from $L^{2}(\mu)$ to $L^{2}(\nu)$ is onto. They consider specifically compactly supported $\mu$ and purely atomic $\nu$. Similarly, problems of equivalent norms in Paley-Wiener spaces are considered in OCS02, LS02,

In a slightly different view, the series of papers by Strichartz Str90, Str93a, Str93b considers a type of Plancherel duality between self-similar measures and a limit of localized Lebesgue measures. As a typical result, Strichartz proves that for a suitable fractal measure $\mu$ on $\mathbb{R}^{d}$ which is "locally $\alpha$-dimensional",

$$
\int_{\mathbb{R}^{d}}|f(x)|^{2} d \mu \simeq \limsup _{R \rightarrow \infty} \frac{1}{R^{d-\alpha}} \int_{B_{0}(R)}|\widehat{f d \mu}(t)|^{2} d \lambda .
$$

Extensions of these results are contained in [MZ09].

Finally, GH03 introduces a concept of continuous frame, that is, a frame which is not a sequence of vectors in a Hilbert space but instead a set of vectors parametrized by a measure space. The dual measure considered in the present paper is a concrete case of Gabardo and Han's definition, where here the vectors are specifically exponential functions.

In recent years there has been a wide range of interest in expanding the classical Fourier analysis to fractal or more general probability measures DHS09, HL08, JP98, DHS09, HL08, IP00, JP98, JKS07, ŁW06, Li07, Str00, Str06, Yua08. One of the central themes of this area of research involves constructive and computational 
bases in $L^{2}(\mu)$, where $\mu$ is a measure which is determined by some self-similarity property. These include classical Fourier bases, as well as wavelet and frame constructions.

We are motivated by questions of Fourier frames for fractal measures DHSW11, DHW11. Specifically, we are motivated by the question of whether the Cantor measure has a Fourier frame. For the middle-third Cantor set, there is a canonical measure $\mu_{3}$ which is supported on the Cantor set-it is known that there is no orthonormal basis of exponentials for $L^{2}\left(\mu_{3}\right)$ JP98. It was shown in DHW11] that there exists a sequence of exponentials which has (relatively) large Beurling dimension CKS08 and forms a Bessel sequence. It is still unknown if there is a Fourier frame for $\mu_{3}$.

In contrast, a Cantor like set with an associated measure $\mu_{4}$ constructed in JP98. does possess an orthonormal basis of exponentials. It was shown in DHW11] that any Fourier frame for $\mu_{4}$ must have the property that the sequence of frequencies must have Beurling dimension at most $\frac{1}{2}$. However, we show in Corollary 4.7 that the integer lattice can be weighted so that a sequence of weighted exponentials forms a frame for $\mu_{4}$, but the sequence of integers which have a non-zero weight has Beurling dimension 1. In the context of dual measures, there exists a sequence of weights $\left\{d_{n}\right\}$ such that $\nu=\sum_{n \in \mathbb{Z}} d_{n} \delta_{n}$ is a dual measure to $\mu_{4}$. We will introduce an appropriate notion of Beurling dimension for measures, and this measure $\nu$ will have dimension $\frac{1}{2}$ as predicted by Theorem 3.8 .

The rest of this paper is organized as follows: we introduce basic definitions next. In section 2 we investigate some general properties about Bessel and frame measures. We demonstrate in Theorem 2.2 that there cannot be any general statement concerning the existence of frame measures. Moreover, we cannot even say that frame measures exist for invariant measures of iterated function systems, which is our motivating example. However, by using convolutions of measures, we show that if one frame measure exists, then in general many frame measures exist (Proposition 2.3). Moreover, if one frame measure exists, then there exists a frame measure which is absolutely continuous with respect to Lebesgue measure and has a smooth Radon-Nikodym derivative (Corollary 2.5). At the other extreme, again if one frame measure exists, then there exists a frame measure which is atomic (Theorem 2.11). As a consequence, if a measure such as $\mu_{3}$ has a frame measure, then it has a weighted Fourier frame (Remark 1.4). Section 3 is devoted to establishing the connections between frame/Bessel measures and Beurling dimension and density. We prove that the Beurling dimension is invariant under convolutions with probability measures, and under discretizations (Theorem 3.4 and Theorem 3.5). Moreover, we obtain that any Bessel measure with positive lower Beurling density is absolutely continuous with respect to the Lebesque measure, and its Radon-Nikodym derivative in $L^{2}$-integrable. The last section of this paper is focused on frame measures for self-affine measures. In Theorem 4.6 we prove that under certain tiling assumptions one can use the Fourier transform of the complementing measure as a weight for the Lebesgue measure and obtain a Plancherel measure for the given fractal measure.

Definition 1.1. Denote by $\delta_{a}$ the Dirac measure at the point $a$. Denote by $e_{t}$, $t \in \mathbb{R}^{d}$, the exponential function

$$
e_{t}(x)=e^{2 \pi i t \cdot x} \quad\left(x \in \mathbb{R}^{d}\right) .
$$


Definition 1.2. A sequence $\left\{x_{n}\right\}_{n=1}^{\infty}$ in a Hilbert space (with inner product $\langle\cdot, \cdot\rangle$ ) is Bessel if there exists a positive constant $B$ such that

$$
\sum_{n=1}^{\infty}\left|\left\langle v, x_{n}\right\rangle\right|^{2} \leq B\|v\|^{2} \text { for all } v .
$$

The sequence is a frame if in addition to being a Bessel sequence there exists a positive constant $A$ such that

$$
A\|v\|^{2} \leq \sum_{n=1}^{\infty}\left|\left\langle v, x_{n}\right\rangle\right|^{2} \leq B\|v\|^{2} .
$$

In this case, $A$ and $B$ are called the lower and upper frame bounds, respectively.

We extend these ideas as follows.

Definition 1.3. We say that a Borel measure $\nu$ is a Bessel measure for $\mu$ if there exists a constant $B>0$ such that for every $f \in L^{2}(\mu)$, we have

$$
\|\widehat{f d \mu}\|_{L^{2}(\nu)}^{2} \leq B\|f\|_{L^{2}(\mu)}^{2} .
$$

We call $B$ a (Bessel) bound for $\nu$. We say the measure $\nu$ is a frame measure for $\mu$ if there exist constants $A, B>0$ such that for every $f \in L^{2}(\mu)$, we have

$$
A\|f\|_{L^{2}(\mu)}^{2} \leq\|\widehat{f d \mu}\|_{L^{2}(\nu)}^{2} \leq B\|f\|_{L^{2}(\mu)}^{2} .
$$

We call $A, B$ (frame) bounds for $\nu$. We call $\nu$ a Plancherel measure if $A=B=1$. We say that a set $\Lambda$ in $\mathbb{R}^{d}$ is a spectrum for $\mu$ if the set $E(\Lambda)=\left\{e_{\lambda}: \lambda \in \Lambda\right\}$ is an orthonormal basis for $L^{2}(\mu)$.

Remark 1.4. Note that, as mentioned previously, if $\left\{e_{\lambda_{n}}: n \in \mathbb{Z}\right\} \in L^{2}(\mu)$ is a frame, then the measure $\nu=\sum_{n \in \mathbb{Z}} \delta_{\lambda_{n}}$ is a frame measure. Conversely, if $\nu$ is purely atomic, i.e. $\nu=\sum_{n \in \mathbb{Z}} d_{n} \delta_{\lambda_{n}}$, and is a frame measure for $\mu$, then $\left\{\sqrt{d_{n}} e_{\lambda_{n}}\right\}$ is a (weighted) Fourier frame for $L^{2}(\mu)$. Indeed, we have

$$
\begin{aligned}
A\|f\|_{L^{2}(\mu)}^{2} \leq\|\widehat{f d \mu}\|_{L^{2}(\nu)}^{2} & =\sum_{n \in \mathbb{Z}} d_{n}\left|\widehat{f d \mu}\left(\lambda_{n}\right)\right|^{2} \\
& =\sum_{n \in \mathbb{Z}}\left|\left\langle f, \sqrt{d_{n}} e_{\lambda_{n}}\right\rangle\right|^{2} \leq B\|f\|_{L^{2}(\mu)}^{2} .
\end{aligned}
$$

Here we require that $\mu$ be a finite Borel measure in order for $e_{\lambda_{n}} \in L^{2}(\mu)$. For the remainder of the paper, we shall assume that $\mu$ is a Borel probability measure, unless stated explicitly otherwise.

\section{Qualitative STructure Results}

In this section we prove some general results concerning Bessel and frame measures $\nu$ for a given measure $\mu$. We begin with a proof that any Bessel measure $\nu$ must be locally finite, i.e. $\nu(K)<\infty$ for all compact subsets $K$ of $\mathbb{R}^{d}$, and hence is $\sigma$-finite. This will allow us the use of the Fubini theorem.

Proposition 2.1. If $\nu$ is a Bessel measure for the measure $\mu$, then there exists a constant $C$ such that $\nu(K) \leq C \max \left\{1, \operatorname{diam}(K)^{d}\right\}$ for any compact subset $K$ of $\mathbb{R}^{d}$. Consequently, $\nu$ is $\sigma$-finite. 
Proof. Since $\widehat{d \mu}(0)=\mu\left(\mathbb{R}^{d}\right)>0$ there exits $\epsilon>0$ and $\delta>0$ such that $|\widehat{d \mu}(x)|^{2} \geq \delta$ for $x \in B(0, \epsilon)$. Then for any $t \in \mathbb{R}^{d}$ we have

$$
\begin{aligned}
B=B\left\|e_{t}\right\|_{L^{2}(\mu)}^{2} & \geq \int\left|\widehat{e_{t} d \mu}(x)\right|^{2} d \nu(x)=\int|\widehat{d \mu}(x-t)|^{2} d \nu(x) \\
& \geq \int_{B(t, \epsilon)}|\widehat{d \mu}(x-t)|^{2} d \nu(x) \geq \nu(B(t, \epsilon)) \delta .
\end{aligned}
$$

Therefore $\nu(B(t, \epsilon)) \leq B / \delta$. This implies that if a compact set has diameter less than $\epsilon$, then its measure is bounded by $B / \delta$. Since any compact set $K$ can be covered by some universal constant times $\operatorname{diam}(K)^{d}$ such balls, the result follows.

Theorem 2.2. There exist finite compactly supported Borel measures that do not admit frame measures.

Proof. Let $\mu=\chi_{[0,1]} d x+\delta_{2}$. Suppose $\nu$ is a frame measure for $\mu$ with frame bounds $A, B>0$. Let $f:=\chi_{\{2\}}$. Then $|\widehat{f d \mu}(t)|=1$ and $\|f\|_{L^{2}(\mu)}=1$. The Bessel bound implies that $\nu(\mathbb{R}) \leq B<\infty$. From this we obtain that for any $\epsilon>0$ there exists $R>0$ such that $\nu\left(\mathbb{R} \backslash B_{0}(R)\right)<\epsilon$.

Now take some $T$ large, arbitrary and let $g(x):=e^{-2 \pi i T x} \chi_{[0,1]}$. We have

$$
|\widehat{g d \mu}(t)|^{2}=\frac{\sin ^{2}(\pi(T+t))}{\pi^{2}(T+t)^{2}} \quad(t \in \mathbb{R}) .
$$

Therefore $|\widehat{g d \mu}(t)|^{2} \leq 1$ for all $t \in \mathbb{R}$, and taking $T \geq 2 R$, we have

$$
|\widehat{g d \mu}(t)|^{2} \leq \frac{1}{\pi^{2}(T-R)^{2}} \text { for all } t \in(-R, R) \text {. }
$$

Then, using the lower frame bound,

$$
\begin{aligned}
A=A\|g\|_{L^{2}(\mu)}^{2} & \leq \int|\widehat{g d \mu}(t)|^{2} d \nu(t)=\int_{B(0, R)}|\widehat{g d \mu}(t)|^{2} d \nu(t)+\int_{\mathbb{R} \backslash B(0, R)}|\widehat{g d \mu}(t)|^{2} d \nu \\
& \leq \frac{1}{\pi^{2}(T-R)^{2}} \cdot \nu(\mathbb{R})+\epsilon .
\end{aligned}
$$

Letting $T \rightarrow \infty$ and $\epsilon \rightarrow 0$ we obtain that $A=0$, a contradiction.

The next proposition shows that once a Bessel/frame measure is present, many others can be constructed.

Proposition 2.3. For fixed positive constants $A$ and $B$, let $\mathcal{B}_{B}(\mu)$ denote the set of all Bessel measures with bound $B$ and let $\mathcal{F}_{A, B}(\mu)$ denote the set of all frame measures with bounds $A, B$. Both sets, while possibly empty, are convex and closed under convolution with Borel probability measures.

Proof. To see that these sets are convex requires just a direct computation. We check that $\mathcal{B}_{B}(\mu)$ is closed under convolution with Borel probability measures; for the set of frame measures the proof in analogous. Take $\nu \in \mathcal{B}_{B}(\mu)$ and let $\rho$ be a Borel probability measure on $\mathbb{R}^{d}$. Take $f \in L^{2}(\mu)$. Then

$$
\begin{gathered}
\int|\widehat{f d \mu}(t)|^{2} d \nu * \rho(t)=\iint|\widehat{f d \mu}(t+s)|^{2} d \nu(t) d \rho(s)=\iint\left|\widehat{e_{-s} f d} \mu(t)\right|^{2} d \nu(t) d \rho(s) \\
\leq \int B\left\|e_{-s} f\right\|_{L^{2}(\mu)}^{2} d \rho(s)=B \int\|f\|_{L^{2}(\mu)}^{2} d \rho(s)=B\|f\|_{L^{2}(\mu)}^{2} .
\end{gathered}
$$


Remark 2.4. The set $\mathcal{B}_{B}(\mu)$ is never empty. One can take $\nu=\sum_{i \in I} c_{i} \delta_{\lambda_{i}}$ for some $\lambda_{i} \in \mathbb{R}^{d}$ and adjust the constants such that $\sum_{i \in I} c_{i} \leq B / \mu\left(\mathbb{R}^{d}\right)^{2}$. Then $\nu \in \mathcal{B}_{B}(\mu)$.

Corollary 2.5. If $\mu$ has a Bessel/frame measure $\nu$, then it has one which is absolutely continuous with respect to the Lebesgue measure and whose Radon-Nikodym derivative is $C^{\infty}$.

Proof. Convoluting $\nu$ with the Lebesgue measure on $[0,1]$ one obtains a Bessel/frame measure which is absolutely continuous. Then, convoluting with $g d t$ where $g \geq 0$ is a compactly supported $C^{\infty}$-function with $\int g(t) d t=1$, one obtains the desired measure.

The next theorem shows that the Bessel/frame property is preserved under approximations that use convolution kernels.

Theorem 2.6. Let $\lambda_{n}$ be an approximate identity, in the sense that $\lambda_{n}$ is a Borel probability measure with the property that $\sup \left\{\|t\|: t \in \operatorname{supp} \lambda_{n}\right\} \rightarrow 0$ as $n \rightarrow \infty$. Suppose $\nu$ is a $\sigma$-finite Borel measure, and suppose $\nu * \lambda_{n}$ are Bessel/frame measures for $\mu$ with uniform bounds, independent of $n$. Then $\nu$ is a Bessel/frame measure.

Proof. We show first that if $f$ is a continuous function on $\mathbb{R}^{d}$, then for any $x \in \mathbb{R}^{d}$,

$$
\int f(x+t) d \lambda_{n}(t) \rightarrow f(x) \text { as } n \rightarrow \infty .
$$

Fix $\epsilon>0$. Since $f$ is continuous at $x$ there exists $\delta>0$ such that $|f(x+t)-f(x)|<\epsilon$ if $\|t\|<\delta$. There exists $n_{\delta}$ such that the support of $\lambda_{n}$ is contained in $B(0, \delta)$ for all $n \geq n_{\delta}$. Then

$$
\begin{aligned}
\left|\int f(x+t) d \lambda_{n}(t)-f(x)\right| & \leq \int|f(x+t)-f(x)| d \lambda_{n}(t)=\int_{B(0, \delta)}|f(x+t)-f(x)| d \lambda_{n}(t) \\
& \leq \int \epsilon d \lambda_{n}=\epsilon .
\end{aligned}
$$

We prove first that $\nu$ is a Bessel measure with the same bound $B$ as $\nu * \lambda_{n}$. Take $f \in L^{2}(\mu)$. Since $\widehat{f d \mu}$ is continuous, we have by Fatou's lemma:

$$
\begin{aligned}
\int|\widehat{f d \mu}(x)|^{2} d \nu(x) & =\int \lim _{n} \int|\widehat{f d \mu}(x+t)|^{2} d \lambda_{n}(t) d \nu(x) \\
& \leq \liminf _{n} \iint|\widehat{f d \mu}(x+t)|^{2} d \lambda_{n}(t) d \nu(x) \\
& =\liminf _{n} \int|\widehat{f d \mu}(y)|^{2} d\left(\nu * \lambda_{n}\right)(y) \\
& \leq B\|f\|_{L^{2}(\mu)}^{2} .
\end{aligned}
$$

To obtain the lower bound we have to show that

$$
\int|\widehat{f d \mu}(x)|^{2} d \nu * \lambda_{n} \rightarrow \int|\widehat{f d \mu}(x)|^{2} d \nu
$$

For this, note first that given $M>0$,

$$
\int_{\{\|x\| \leq M\}} \int|\widehat{f d \mu}(x+t)|^{2} d \lambda_{n}(t) d \nu(x) \rightarrow \int_{\{\|x\| \leq M\}}|\widehat{f d \mu(x)}|^{2} d \nu(x) .
$$


This follows from Lebesgue's dominated convergence theorem, since $|\widehat{f d \mu}|$ is bounded (more precisely $\|\widehat{f d \mu}\|_{\infty} \leq\|f\|_{L^{1}(\mu)}$ ), and since $\nu(\{\|x\| \leq M\})<\infty$, by Proposition 2.1.

Secondly, given $\delta>0$ we will find $M>0$ such that for $n$ large enough

$$
\int_{\{\|x\| \geq M\}} \int|\widehat{f d \mu}(x+t)|^{2} d \lambda_{n}(t) d \nu(x)<\delta \text { and } \int_{\{\|x\| \geq M\}}|\widehat{f d \mu(x)}|^{2} d \nu(x)<\delta .
$$

We have

$$
\int_{\{\|x\| \geq M\}}|\widehat{f d \mu}(x+t)|^{2} d \nu(x)=\int \chi_{\{\|x\| \geq M\}}\left|\widehat{e_{-t} f d} \mu(x)\right|^{2} d \nu(x) .
$$

Since $\mu$ is compactly supported, for $t$ small enough, we have that $e_{-t} f$ is close to $f$ in $L^{2}(\mu)$. Since $\nu$ is a Bessel measure we obtain that $\widehat{e_{-t} f d} \mu$ is close to $\widehat{f d \mu}$ in $L^{2}(\nu)$. Multiplying by the characteristic function, we get that $\chi_{\{\|x\| \geq M\}} \widehat{e_{-t} f d} \mu(x)$ is close to $\chi_{\{\|x\| \geq M\}} \widehat{f d \mu}$ in $L^{2}(\nu)$. Since $\widehat{f d \mu}$ is in $L^{2}(\nu)$ we can find $M$ such that

$$
\int \chi_{\{\|x\| \geq M\}}|\widehat{f d \mu}(x)|^{2} d \nu(x)<\delta / 2 .
$$

Then, for $t$ small enough,

$$
\int \chi_{\{\|x\| \geq M\}}\left|\widehat{e_{-t} f d} \mu(x)\right|^{2} d \nu(x)<\delta .
$$

Pick $n$ large enough so that inequality (2.4) holds for all $t$ in the support of $\lambda_{n}$. Then integrating inequality (2.4) with respect to $\lambda_{n}$, we obtain

$$
\iint_{\{\|x\| \geq M\}}|\widehat{f d \mu}(x+t)|^{2} d \nu(x) d \lambda_{n}(t)<\delta .
$$

Inequality (2.3) follows by an application of Fubini's theorem.

The limit in (2.2) follows by splitting the integral into two regions where $\|x\| \leq M$ or $\|x\| \geq M$. Then the lower bound follows from (2.2).

In the following three propositions we present a general way of constructing Bessel/frame measures for a given measure. The idea is that if a convolution of two measures admits a Bessel measure, then one can use the Fourier transform of one of the measures in the convolution as a weight for the Bessel measure to obtain a Bessel measure for the other measure in the convolution. The same is true for frame measures, but with certain restrictions.

Proposition 2.7. Let $\mu, \mu^{\prime}$ be two Borel probability measures. For $f \in L^{1}(\mu)$, the measure $(f d \mu) * \mu^{\prime}$ is absolutely continuous w.r.t. $\mu * \mu^{\prime}$. We denote by $P_{\mu, \mu^{\prime}} f$ or $P f$ the Radon-Nykodim derivative:

$$
P f=\frac{(f d \mu) * \mu^{\prime}}{d\left(\mu * \mu^{\prime}\right)} .
$$

Proof. Take a Borel set $E$ such that $\mu * \mu^{\prime}(E)=0$. Then

$$
0=\iint \chi_{E}(x+y) d \mu(x) d \mu^{\prime}(y)=\int \mu(E-y) d \mu^{\prime}(y) .
$$

This implies that $\mu(E-y)=0$ for $\mu^{\prime}$-a.e. $y$. 
Then

$$
\begin{aligned}
\iint \chi_{E}(x+y) f d \mu(x) d \mu^{\prime}(y) & =\iint \chi_{E}(x+y) f(x) d \mu(x) d \mu^{\prime}(y) \\
& =\iint \chi_{E-y}(x) f(x) d \mu(x) d \mu^{\prime}(y)=0 .
\end{aligned}
$$

So $(f d \mu) * \mu^{\prime}(E)=0$.

Proposition 2.8. Let $\mu$ and $\mu^{\prime}$ be probability measures and $1 \leq p \leq \infty$. If $f \in$ $L^{p}(\mu)$, then the function $P f$ is also in $L^{p}\left(\mu * \mu^{\prime}\right)$ and

$$
\|P f\|_{L^{p}\left(\mu * \mu^{\prime}\right)} \leq\|f\|_{L^{p}(\mu)} .
$$

Proof. For $p=\infty$, let $g \in L^{1}\left(\mu * \mu^{\prime}\right)$. Then

$$
\begin{aligned}
\left|\int g P f d \mu * \mu^{\prime}\right| & =\left|\int g d\left((f d \mu) * \mu^{\prime}\right)\right|=\left|\iint g(x+y) f(x) d \mu(x) d \mu^{\prime}(y)\right| \\
& \leq\|f\|_{\infty}\left|\iint\right| g(x+y)|d \mu(x) d \mu(y)|=\|f\|_{\infty} \int|g| d\left(\mu * \mu^{\prime}\right) .
\end{aligned}
$$

Suppose $\|P f\|_{\infty}>\|f\|_{\infty}$. Then for $\epsilon>0$ small, the set $A:=\{x:|P f(x)| \geq$ $\left.\|f\|_{\infty}+\epsilon\right\}$ has positive $\mu * \mu^{\prime}$-measure. Let $g:=\frac{|P f|}{P f} \chi_{A}$. We have that $g \in L^{1}\left(\mu * \mu^{\prime}\right)$ and

$\left|\int g P f d \mu * \mu^{\prime}\right|=\int_{A}|P f| d \mu * \mu^{\prime} \geq\left(\|f\|_{\infty}+\epsilon\right) \mu * \mu^{\prime}(A)=\left(\|f\|_{\infty}+\epsilon\right) \int|g| d \mu * \mu^{\prime}$.

This contradicts the previous computation.

For $p=1$, take $g(x)=|P f(x)| / P f(x)$ if $P f(x) \neq 0$ and $g(x)=0$ otherwise. Then

$$
\begin{aligned}
& \int|P f| d \mu * \mu^{\prime}=\int g P f d \mu * \mu^{\prime}=\int g d\left((f d \mu) * \mu^{\prime}\right) \\
&=\iint g(x+y) f(x) d \mu(x) d \mu(y) \leq \iint|f(x)| d \mu(x) d \mu(y)=\|f\|_{L^{1}(\mu)} .
\end{aligned}
$$

For $1<p<\infty$ the inequality follows from the Riesz-Thorin interpolation theorem.

Proposition 2.9. Let $\mu, \mu^{\prime}$ be probability measures. Assume that $\mu * \mu^{\prime}$ has a Bessel measure $\nu$. Then $\left|\hat{\mu}^{\prime}\right|^{2} d \nu$ is a Bessel measure for $\mu$ with the same bound.

If in addition $\nu$ is a frame measure for $\mu * \mu^{\prime}$ with bounds $A$ and $B$, and $c\|f\|_{L^{2}(\mu)}^{2} \leq\|P f\|_{L^{2}\left(\mu * \mu^{\prime}\right)}^{2}$ for all $f \in L^{2}(\mu)$, then $\left|\hat{\mu}^{\prime}\right|^{2} d \nu$ is a frame measure for $\mu$ with bounds $c A$ and $B$.

Proof. Take $f \in L^{2}(\mu)$. Then

$$
\begin{aligned}
\int|\widehat{(f d \mu)}|^{2} \cdot\left|\widehat{\mu}^{\prime}\right|^{2} d \nu & =\int \mid\left(\left.\widehat{f \mu) * \mu^{\prime}}\right|^{2} d \nu=\int\left|\left(P \widehat{f d \mu *} \mu^{\prime}\right)\right|^{2} d \nu\right. \\
& \geq A\|P f\|_{L^{2}\left(\mu * \mu^{\prime}\right)}^{2} \geq c A\|f\|_{L^{2}(\mu)}^{2} .
\end{aligned}
$$

The upper bound follows from Proposition 2.8.

Next we prove some stability results. 
Theorem 2.10. Let $\mu$ be a compactly supported Borel probability measure. If $\nu$ is a Bessel measure for $\mu$, then for any $r>0$ there exists a constant $D>0$ such that

$$
\int \sup _{|y| \leq r}|\widehat{f d \mu}(x+y)|^{2} d \nu(x) \leq D\|f\|_{L^{2}(\mu)}^{2}, \text { for all } f \in L^{2}(\mu) .
$$

If $\nu$ is a frame measure for $\mu$, then there exist constants $\delta>0$ and $C>0$ such that

$$
C\|f\|_{L^{2}(\mu)}^{2} \leq \int \inf _{|y| \leq \delta}|\widehat{f d \mu}(x+y)|^{2} d \nu(x), \text { for all } f \in L^{2}(\mu) .
$$

Proof. Let $\epsilon: \mathbb{R}^{d} \rightarrow \mathbb{R}^{d}$ be some Borel measurable function, such that $|\epsilon(x)| \leq r$ for all $x \in \mathbb{R}^{d}$. Take $f \in L^{2}(\mu)$ and $x \in \mathbb{R}^{d}$. Let $u:=\epsilon(x) /|\epsilon(x)|$ and

$$
g_{x}(t):=\widehat{f d \mu}(x+t u) \quad(t \in \mathbb{R}) .
$$

The function $g$ is analytic and its derivatives are

$$
g_{x}^{(k)}(t)=\int e^{-2 \pi i(x+t u) \cdot y}(-2 \pi i u \cdot y)^{k} f(y) d \mu(y)=\left((-2 \pi i u \cdot y)^{k} f \widehat{)}(x+t u) .\right.
$$

Writing the Taylor expansion at 0 we have

$$
\left|g_{x}(|\epsilon(x)|)-g_{x}(0)\right|^{2}=\left.\left.\left|\sum_{k=1}^{\infty} \frac{g_{x}^{(k)}(0)}{k !}\right| \epsilon(x)\right|^{k}\right|^{2},
$$

and using the Cauchy-Schwartz inequality

$$
\leq \sum_{k=1}^{\infty} \frac{\left|g_{x}^{(k)}(0)\right|^{2}}{k !} \sum_{k=1}^{\infty} \frac{|\epsilon(x)|^{2 k}}{k !}=\sum_{k=1}^{\infty} \frac{\left|g_{x}^{(k)}(0)\right|^{2}}{k !} \cdot\left(e^{|\epsilon(x)|^{2}}-1\right) \leq\left(e^{r^{2}}-1\right) \sum_{k=1}^{\infty} \frac{\left|g_{x}^{(k)}(0)\right|^{2}}{k !} \text {. }
$$

We use the Bessel bound and obtain

$$
\begin{aligned}
\int\left|g_{x}^{(k)}(0)\right|^{2} d \nu(x) & =\int \mid\left(\left.(-2 \pi i u \cdot y)^{k} f \widehat{)}(x)\right|^{2} d \nu(x) \leq B\left\|(-2 \pi i u \cdot y)^{k} f(y)\right\|_{L^{2}(\mu)}^{2}\right. \\
& \leq(2 \pi M)^{2 k} B\|f\|_{L^{2}(\mu)}^{2},
\end{aligned}
$$

where the constant $M$ is chosen such that the support of $\mu$ is contained in the ball $|x| \leq M$. Then

$$
\begin{aligned}
& \int|\widehat{f d \mu}(x+\epsilon(x))-\widehat{f d \mu}(x)|^{2} d \nu(x) \\
& \quad=\int\left|g_{x}(|\epsilon(x)|)-g_{x}(0)\right|^{2} d \nu(x) \leq\left(e^{r^{2}}-1\right) \int \sum_{k=1}^{\infty} \frac{\left|g_{x}^{(k)}(0)\right|^{2}}{k !} d \nu(x) \\
& \quad=\left(e^{r^{2}}-1\right) \sum_{k=1}^{\infty} \frac{1}{k !} \int\left|g_{x}^{(k)}(0)\right|^{2} d \nu(x) \leq\left(e^{r^{2}}-1\right) \sum_{k=1}^{\infty} \frac{1}{k !}(2 \pi M)^{2 k} B\|f\|_{L^{2}(\mu)^{2}}^{2} \\
& \quad=B\left(e^{r^{2}}-1\right)\left(e^{(2 \pi M)^{2}}-1\right)\|f\|_{L^{2}(\mu)}^{2} .
\end{aligned}
$$

Then, by Minkowski's inequality,

$$
\begin{aligned}
\left(\int|\widehat{f d \mu}(x+\epsilon(x))|^{2} d \nu(x)\right)^{\frac{1}{2}} \leq & \left(\int|\widehat{f d \mu}(x)|^{2} d \nu(x)\right)^{\frac{1}{2}} \\
& +\left(\int|\widehat{f d \mu}(x+\epsilon(x))-\widehat{f d \mu}(x)|^{2} d \mu(x)\right)^{\frac{1}{2}} \\
\leq & \left(B^{\frac{1}{2}}+\left(B\left(e^{r^{2}}-1\right)\left(e^{(2 \pi M)^{2}}-1\right)\right)^{\frac{1}{2}}\right)\|f\|_{L^{2}(\mu)} .
\end{aligned}
$$


Taking $\epsilon(x)$ such that $|\widehat{f d \mu}(x+\epsilon(x))|^{2}=\sup _{|y| \leq r}|\widehat{f d \mu}(x+y)|^{2}$, we obtain (2.6). Such a function $\epsilon$ is Borel measurable since the function $\widehat{f d \mu}$ is continuous, and analytic in each variable.

For (2.7), take $\delta=r>0$ small enough such that

$$
C:=A^{\frac{1}{2}}-\left(B\left(e^{r^{2}}-1\right)\left(e^{(2 \pi M)^{2}}-1\right)\right)^{\frac{1}{2}}>0,
$$

where $A$ is the lower frame bound for $\nu$.

Then, by Minkowski's inequality,

$$
\begin{aligned}
\left(\int|\widehat{f d \mu}(x+\epsilon(x))|^{2} d \nu(x)\right)^{\frac{1}{2}} \geq & \left(\int|\widehat{f d \mu}(x)|^{2} d \nu(x)\right)^{\frac{1}{2}} \\
& -\left(\int|\widehat{f d \mu}(x+\epsilon(x))-\widehat{f d \mu}(x)|^{2} d \mu(x)\right)^{\frac{1}{2}} \\
\geq & \left(A^{\frac{1}{2}}-\left(B\left(e^{r^{2}}-1\right)\left(e^{(2 \pi M)^{2}}-1\right)\right)^{\frac{1}{2}}\right)\|f\|_{L^{2}(\mu)} .
\end{aligned}
$$
(2.7).

Take $\epsilon(x)$ such that $|\widehat{f d \mu}(x+\epsilon(x))|^{2}=\inf _{|y| \leq r}|\widehat{f d \mu}(x+y)|^{2}$, and we obtain

Using this stability of frame measures we can prove that a certain form of discretization will produce atomic frame measures from a general frame measure.

Theorem 2.11. If a measure $\mu$ has a Bessel/frame measure $\nu$, then it also has an atomic one. More precisely, let $Q=[0,1)^{d}$ and $r>0$. If $\nu$ is a Bessel measure for $\mu$ and $\left(x_{k}\right)_{k \in \mathbb{Z}^{d}}$ is a set of points such that $x_{k} \in r(k+Q)$ for all $k \in \mathbb{Z}^{d}$, then

$$
\nu^{\prime}:=\sum_{k \in \mathbb{Z}^{d}} \nu(r(k+Q)) \delta_{x_{k}}
$$

is a Bessel measure for $\mu$. We call $\nu^{\prime}$ a discretization of the measure $\nu$.

If $\nu$ is a frame measure for $\mu$ and $r$ is small enough, then the measure $\nu^{\prime}$ defined above is a frame measure for $\mu$.

Proof. Define $\epsilon(x)=x_{k}-x$ if $x \in r(k+Q)$. Then, $|\epsilon(x)| \leq r \sqrt{d}=: r^{\prime}$ and for all $f \in L^{2}(\mu)$,

$$
\begin{aligned}
\int|\widehat{f d \mu}(x+\epsilon(x))|^{2} d \nu(x) & =\sum_{k \in \mathbb{Z}^{d}} \int_{r(k+Q)}\left|\widehat{f d \mu}\left(x_{k}\right)\right|^{2} d \nu(x) \\
& =\sum_{k \in \mathbb{Z}^{d}} \nu(r(k+Q))\left|\widehat{f d \mu}\left(x_{k}\right)\right|^{2}
\end{aligned}
$$

But since

$$
\begin{aligned}
\int \inf _{|y| \leq r^{\prime}}|\widehat{f d \mu}(x+y)|^{2} d \nu(x) & \leq \int|\widehat{f d \mu}(x+\epsilon(x))|^{2} d \nu(x) \\
& \leq \int \sup _{|y| \leq r^{\prime}}|\widehat{f d \mu}(x+y)|^{2} d \nu(x),
\end{aligned}
$$

everything follows from Theorem 2.10 .

Corollary 2.12. If $\nu$ is a frame measure for $\mu$ and $r>0$ is sufficiently small, then $\left\{c_{k} e_{x_{k}}: k \in \mathbb{Z}^{d}\right\}$ is a weighted Fourier frame for $\mu$, where $x_{k} \in r(k+Q)$ and $c_{k}=\sqrt{\nu(r(k+Q))}$. 
Proof. The measure $\nu^{\prime}=\sum_{k \in \mathbb{Z}^{d}} c_{k}^{2} \delta_{x_{k}}$ is a frame measure for $\mu$, so for any $f \in L^{2}(\mu)$ we have

$$
A\|f\|_{\mu}^{2} \leq \int|\widehat{f d \mu}(t)|^{2} d \nu^{\prime}=\sum_{k \in \mathbb{Z}^{d}} c_{k}^{2}\left|\widehat{f d \mu}\left(x_{k}\right)\right|^{2}=\sum_{k \in \mathbb{Z}^{d}}\left|\left\langle f, c_{k} e_{x_{k}}\right\rangle\right|^{2} \leq B\|f\|_{\mu}^{2} .
$$

\section{Beurling Dimension}

In DHSW11, Beurling dimension (of sequences) as defined in CKS08 is used to provide a partial characterization of Bessel sequences of exponentials. We extend the definition of Beurling dimension to measures, and using this definition we obtain similar results concerning Bessel measures. Specifically, we show that any Bessel measure for $\mu$ must have Beurling dimension which is sufficiently small, depending upon a certain property that $\mu$ may possess. We begin with some basic properties (including certain invariances which will be useful later) of Beurling dimension of measures.

Definition 3.1. Let $Q$ be the unit cube $Q=[0,1)^{d}$. For a locally finite measure $\nu$ and $\alpha \geq 0$ we define the $\alpha$-upper Beurling density by

$$
\mathcal{D}_{\alpha}(\nu):=\limsup _{R \rightarrow \infty} \sup _{x \in \mathbb{R}^{d}} \frac{\nu(x+R Q)}{R^{\alpha}} .
$$

We define the (upper) Beurling dimension of $\nu$ by

$$
\operatorname{dim} \nu:=\sup \left\{\alpha \geq 0: \mathcal{D}_{\alpha}(\nu)=\infty\right\} .
$$

Proposition 3.2. If $\nu$ is a locally finite Borel measure, then

$$
\mathcal{D}_{\alpha}(\nu)=\infty \text { for } \alpha<\operatorname{dim} \nu \text { and } \mathcal{D}_{\alpha}(\nu)=0 \text { for } \alpha>\operatorname{dim} \nu .
$$

In particular,

$$
\operatorname{dim} \nu=\inf \left\{\alpha \geq 0: \mathcal{D}_{\alpha}(\nu)=0\right\} .
$$

Proof. Take $\alpha<\operatorname{dim} \nu$. Then there exists $\alpha^{\prime}>\alpha$ such that $\mathcal{D}_{\alpha^{\prime}}(\nu)=\infty$. Then

$$
\mathcal{D}_{\alpha}(\nu)=\limsup _{R \rightarrow \infty} \sup _{x \in \mathbb{R}} \frac{\nu(x+R Q)}{R^{\alpha}} \geq \limsup _{R \rightarrow \infty} \sup _{x \in \mathbb{R}} \frac{\nu(x+R Q)}{R^{\alpha^{\prime}}}=\infty .
$$

If $\alpha>\operatorname{dim} \nu$, then take $\alpha^{\prime}$ such that $\operatorname{dim} \nu<\alpha^{\prime}<\alpha$. From the definition of $\operatorname{dim} \nu$ we have that $\mathcal{D}_{\alpha^{\prime}}(\nu)<\infty$. Then

$$
\begin{gathered}
\mathcal{D}_{\alpha}(\nu)=\limsup _{R \rightarrow \infty} \sup _{x \in \mathbb{R}} \frac{\nu(x+R Q)}{R^{\alpha}}=\limsup _{R \rightarrow \infty} \sup _{x \in \mathbb{R}} \frac{\nu(x+R Q)}{R^{\alpha^{\prime}}} \cdot \frac{R^{\alpha^{\prime}}}{R^{\alpha}} \\
\leq \limsup _{R \rightarrow \infty} \sup _{x \in \mathbb{R}} \frac{\nu(x+R Q)}{R^{\alpha^{\prime}}} \cdot \limsup _{R \rightarrow \infty} \frac{R^{\alpha^{\prime}}}{R^{\alpha}}=\mathcal{D}_{\alpha^{\prime}}(\nu) \cdot 0=0 .
\end{gathered}
$$

Proposition 3.3. The Beurling dimension can be computed by replacing the set $Q$ by any set $O$ that is bounded and has an interior point. 
Proof. There exist $a, b \in \mathbb{R}^{d}$ and $r_{0}, R_{0}>0$ such that

$$
a+r_{0} O \subset Q \subset b+R_{0} O .
$$

We have

$$
\begin{aligned}
& \limsup _{R} \sup _{x} \frac{\nu\left(x+R\left(a+r_{0} O\right)\right)}{R^{\alpha}}=\limsup _{R} \sup _{y} \frac{\nu\left(y+R r_{0} O\right)}{R^{\alpha}} \\
& =\frac{1}{r_{0}^{-\alpha}} \limsup _{R^{\prime}} \sup _{y} \frac{\nu\left(y+R^{\prime} O\right)}{R^{\prime \alpha}} \text {. }
\end{aligned}
$$

Therefore the two quantities involving limsup sup... are simultaneously 0 or $\infty$. Similarly,

$$
\limsup _{R} \sup _{x} \frac{\nu\left(x+R\left(b+R_{0} O\right)\right)}{R^{\alpha}}=\frac{1}{R_{0}^{-\alpha}} \limsup \sup _{R^{\prime}} \frac{\nu\left(y+R^{\prime} O\right)}{R^{\prime \alpha}} .
$$

Since we have

$$
\begin{aligned}
\limsup \sup _{x} \frac{\nu\left(x+R\left(a+r_{0} O\right)\right)}{R^{\alpha}} & \leq \limsup _{R} \sup _{x} \frac{\nu(x+R Q)}{R^{\alpha}} \\
& \leq \limsup \sup _{x} \frac{\nu\left(x+R\left(b+R_{0} O\right)\right)}{R^{\alpha}}
\end{aligned}
$$

we see that the limsup sup ... involving $Q$ and the one involving $O$ are at the same time 0 or $\infty$. The result then follows from Proposition 3.2 .

Theorem 3.4. Let $\nu$ be a locally finite measure and $\rho$ a Borel probability measure. Then

$$
\operatorname{dim} \nu * \rho=\operatorname{dim} \nu
$$

In other words, the Beurling dimension is invariant under convolution with probability measures.

Proof. Let $\alpha_{0}:=\operatorname{dim} \nu$ and take $\alpha>\alpha_{0}$. Then $\mathcal{D}_{\alpha}(\nu)=0$. Therefore, for any $\epsilon>0$, there exists $R$ as large as we want such that

$$
\frac{\nu(x+R Q)}{R^{\alpha}}<\epsilon \text { for all } x \in \mathbb{R}^{d}
$$

Then, for all $x \in \mathbb{R}^{d}$,

$$
\nu * \rho(x+R Q)=\int \nu(x+R Q-t) d \rho(t) \leq \int \epsilon R^{\alpha} d \rho(t)=\epsilon R^{\alpha} .
$$

This implies that

$$
\sup _{x \in \mathbb{R}^{d}} \frac{\nu * \rho(x+R Q)}{R^{\alpha}} \leq \epsilon,
$$

and since $R$ can be taken arbitrarily large, we obtain that $\mathcal{D}_{\alpha}(\nu * \rho)=0$ so $\operatorname{dim} \nu * \rho \leq$ $\alpha_{0}$.

Now take $\alpha<\alpha_{0}$. We have $\mathcal{D}_{\alpha}(\nu)=\infty$. We use Proposition 3.3 with $O$ as the unit ball. Given $M>0$ there exists $R$ as large as we want such that

$$
\sup _{x \in \mathbb{R}^{d}} \frac{\nu(x+R O)}{R^{\alpha}}>M .
$$

This means that there exists $a \in \mathbb{R}^{d}$ such that

$$
\nu(a+R O) \geq M R^{\alpha} .
$$


Since $\rho\left(\mathbb{R}^{d}\right)=1$ we can pick $R$ large enough so that $\rho(R O) \geq \frac{1}{2}$. Then for any $t \in \mathbb{R}^{d}$ with $|t|<R$ we have $a-t+2 R O \supset a+R O$, and we have

$$
\begin{gathered}
\nu * \rho(a+2 R O)=\int \nu(a-t+2 R O) d \rho(t) \geq \int_{|t|<R} \nu(a-t+2 R O) d \rho(t) \\
\geq \int_{|t|<R} \nu(a+R O) d \rho(t) \geq M R^{\alpha} \rho(R O) \geq \frac{M}{2} R^{\alpha} .
\end{gathered}
$$

Therefore

$$
\sup _{x \in \mathbb{R}^{d}} \frac{\nu * \rho(x+2 R O)}{(2 R)^{\alpha}} \geq \frac{M}{2 \cdot 2^{\alpha}}
$$

Since $M$ is arbitrary, this implies that $\mathcal{D}_{\alpha}(\nu * \rho)=\infty$. Hence $\operatorname{dim} \nu * \rho \geq \alpha_{0}$, and the result follows.

Theorem 3.5. Let $\nu$ be a locally finite Borel measure and $r>0$. For each $k \in \mathbb{Z}^{d}$, let $x_{k}$ be a point in $r(k+Q)$. Define the measure

$$
\nu^{\prime}=\sum_{k \in \mathbb{Z}^{d}} \nu(r(k+Q)) \delta_{x_{k}} .
$$

Then

$$
\operatorname{dim} \nu^{\prime}=\operatorname{dim} \nu
$$

In other words, the Beurling dimension is invariant under discretization.

Proof. Take $\alpha>\operatorname{dim} \nu$. Then, given $\epsilon>0$, for $R$ large enough

$$
\frac{\nu(x+R Q)}{R^{\alpha}}<\epsilon \text { for all } x \in \mathbb{R}^{d} .
$$

Take $x \in \mathbb{R}^{d}$ to be arbitrary. We have

$$
\nu^{\prime}(x+R Q)=\sum_{k: x_{k} \in x+R Q} \nu(r(k+Q))=\nu\left(\bigcup_{x_{k} \in x+R Q} r(k+Q)\right) .
$$

Since $x_{k} \in(x+R Q) \cap(r(k+Q))$ we see that the union of cubes $r(k+Q)$ of side $r$ in (3.5) intersecting the cube $x+R Q$ of side $R$ is contained in a cube of side $R+2 r$, which we call $x^{\prime}+(R+2 r) Q$. Then

$$
\frac{\nu^{\prime}(x+R Q)}{R^{\alpha}} \leq \frac{\nu\left(x^{\prime}+(R+2 r) Q\right)}{R^{\alpha}}=\frac{\nu\left(x^{\prime}+(R+2 r) Q\right)}{(R+2 r)^{\alpha}} \cdot \frac{(R+2 r)^{\alpha}}{R^{\alpha}}<\epsilon \cdot 2
$$

for $R$ large enough (independent of $x$ ). Then

$$
\sup _{x \in \mathbb{R}^{d}} \frac{\nu^{\prime}(x+R Q)}{R^{\alpha}} \leq 2 \epsilon
$$

for $R$ large, so $\operatorname{dim} \nu^{\prime} \leq \alpha$ and $\operatorname{dim} \nu^{\prime} \leq \operatorname{dim} \nu$.

Now take $\alpha<\operatorname{dim} \nu$. Given $M>0$ we can find $R$ as large as we want and $x \in \mathbb{R}^{d}$ such that

$$
\frac{\nu(x+R Q)}{R^{\alpha}} \geq M .
$$

The cube $x+R Q$ is contained in the union $U$ of the cubes $r(k+Q)$ that intersect it. This union $U$ is contained in some cube of side $R+2 r$, say $x^{\prime}+(R+2 r) Q$. 
We have

$$
\begin{aligned}
\nu^{\prime}\left(x^{\prime}+(R+2 r) Q\right) & =\sum_{x_{k} \in x^{\prime}+(R+2 r) Q} \nu(r(k+Q))=\nu\left(\bigcup_{x_{k} \in x^{\prime}+(R+2 r) Q} r(k+Q)\right) \\
& \geq \nu\left(\bigcup_{r(k+Q) \subset x^{\prime}+(R+2 r) Q} r(k+Q)\right) \geq \nu(U) \geq \nu(x+R Q) .
\end{aligned}
$$

Then

$$
\frac{\nu^{\prime}(x+(R+2 r) Q)}{(R+2 r)^{\alpha}} \geq \frac{\nu(x+R Q)}{R^{\alpha}} \cdot \frac{R^{\alpha}}{(R+2 r)^{\alpha}} \geq \frac{M}{2} .
$$

This proves that

$$
\sup _{x \in \mathbb{R}^{d}} \frac{\nu^{\prime}(x+(R+2 r))}{(R+2 r)^{\alpha}} \geq \frac{M}{2},
$$

so $\mathcal{D}_{\alpha}\left(\nu^{\prime}\right)=\infty$ and $\operatorname{dim} \nu^{\prime} \geq \operatorname{dim} \nu$.

We now establish some upper bounds on the Beurling dimension of Bessel measures. The following result is true for any Borel measure $\mu$. Subsequent results (e.g. Theorems 3.8 and 4.3) refine this basic result based on whether $\mu$ has additional structure.

Theorem 3.6. If $\nu$ is a Bessel measure for a Borel measure $\mu$ on $\mathbb{R}^{d}$, then $\operatorname{dim} \nu \leq$ $d$.

Proof. We apply Proposition 3.3 using the closed unit cube $\bar{Q}$ to estimate $\operatorname{dim} \nu$. If $\alpha>d$, we have

$$
\begin{aligned}
\mathcal{D}_{\alpha}(\nu) & =\limsup _{R \rightarrow \infty} \sup _{x \in \mathbb{R}^{d}} \frac{\nu(x+R \bar{Q})}{R^{\alpha}} \\
& \leq \limsup _{R \rightarrow \infty} \frac{C R^{d}}{R^{\alpha}} \\
& =0,
\end{aligned}
$$

where the inequality follows from Proposition 2.1 .

Definition 3.7. We say that a Borel measure $\mu$ is occasionally- $\alpha$-dimensional if there exist a sequence of Borel subsets $E_{n}$ and some constants $c_{1}, c_{2}>0$ such that $\operatorname{diam}\left(E_{n}\right)$ decreases to 0 as $n \rightarrow \infty$,

$$
\begin{gathered}
\sup _{n} \frac{\operatorname{diam}\left(E_{n}\right)}{\operatorname{diam}\left(E_{n+1}\right)}<\infty, \\
c_{1} \operatorname{diam}\left(E_{n}\right)^{\alpha} \leq \mu\left(E_{n}\right) \leq c_{2} \operatorname{diam}\left(E_{n}\right)^{\alpha} \quad(n \geq 0)
\end{gathered}
$$

Theorem 3.8. Let $\mu$ be an occasionally- $\alpha$-dimensional measure and suppose $\nu$ is a Bessel measure for $\mu$. Then $\mathcal{D}_{\alpha}(\nu)<\infty$ and so $\operatorname{dim}(\nu) \leq \alpha$.

Proof. Let $E_{n}$ be a sequence of sets as in Definition 3.7. Let $\epsilon_{n}:=\operatorname{diam}\left(E_{n}\right)$ and pick $a_{n} \in E_{n}$. Choose $\delta>0$ such that

$$
\left|e^{2 \pi i y}-1\right| \leq \frac{1}{2} \text { if }|y| \leq \delta
$$


Let $f_{n}:=\chi_{E_{n}}$. We have, for $|t| \leq \frac{\delta}{\epsilon_{n}}$,

$$
\begin{aligned}
\left|\widehat{f_{n} d \mu}(t)-e^{2 \pi i t \cdot a_{n}} \widehat{f_{n} d \mu}(0)\right| & =\left|\int_{E_{n}}\left(e^{-2 \pi i t \cdot x}-e^{2 \pi i t \cdot a_{n}}\right) d \mu(x)\right| \\
& \leq \int_{E_{n}}\left|e^{2 \pi i t \cdot a_{n}}\left(e^{-2 \pi i t \cdot\left(x-a_{n}\right)}-1\right)\right| d \mu(x) \\
& \leq \int_{E_{n}} \frac{1}{2} d \mu=\frac{1}{2} \mu\left(E_{n}\right),
\end{aligned}
$$

since $\left|x-a_{n}\right|<\operatorname{diam}\left(E_{n}\right)=\epsilon_{n}$ for $x \in E_{n}$, so $\left|t \cdot\left(x-a_{n}\right)\right| \leq \frac{\delta}{\epsilon_{n}} \epsilon_{n}=\delta$.

Also,

$$
\left|e^{2 \pi i t \cdot a_{n}} \widehat{f_{n} d \mu}(0)\right|=\mu\left(E_{n}\right) .
$$

Then

$$
\left|\widehat{f_{n} d \mu}(t)\right| \geq\left|e^{2 \pi i t \cdot a_{n}} \widehat{f_{n} d \mu}(0)\right|-\left|\widehat{f_{n} d \mu}(t)-e^{2 \pi i t \cdot a_{n}} \widehat{f_{n} d \mu}(0)\right| \geq \frac{1}{2} \mu\left(E_{n}\right) \geq \frac{c_{1}}{2} \epsilon_{n}^{\alpha},
$$

for all $|t| \leq \frac{\delta}{\epsilon_{n}}$.

Let $g_{n}:=\frac{f_{n}}{\mu\left(E_{n}\right)^{1 / 2}}$. Then $\left\|g_{n}\right\|_{L^{2}\left(\mu_{n}\right)}=1$ and

$$
\left|\widehat{g_{n} d \mu}(t)\right|^{2} \geq \frac{c_{1}^{2}}{4} \epsilon_{n}^{2 \alpha} \cdot \frac{1}{\mu\left(E_{n}\right)} \geq \frac{c_{1}^{2} \epsilon_{n}^{2 \alpha}}{4 c_{2} \epsilon_{n}^{\alpha}}=: C \epsilon_{n}^{\alpha},
$$

for all $|t| \leq \frac{\delta}{\epsilon_{n}}$, and $C>0$.

Apply the Bessel inequality to the function $e^{2 \pi i a \cdot x} g_{n}(x)$ : for all $a \in \mathbb{R}^{d}$,

$$
B \geq \int\left|\widehat{e_{a} \widehat{g_{n} d} \mu}\right|^{2} d \nu \geq \int_{B\left(a, \frac{\delta}{\epsilon_{n}}\right)}\left|\widehat{g_{n} d \mu}(t-a)\right|^{2} d \nu(t) \geq C \epsilon_{n}^{\alpha} \nu\left(B\left(a, \frac{\delta}{\epsilon_{n}}\right)\right) .
$$

Then

$$
\frac{1}{\delta^{\alpha}} \frac{B}{C} \geq \frac{\nu\left(B\left(a, \frac{\delta}{\epsilon_{n}}\right)\right)}{\left(\frac{\delta}{\epsilon_{n}}\right)^{\alpha}}
$$

Now take $M \geq \epsilon_{n} / \epsilon_{n+1}$ for all $n$ (according to (3.6)), and pick $R>0$ large. Let $n$ be such that $\frac{\delta}{\epsilon_{n}} \leq R \leq \frac{\delta}{\epsilon_{n+1}}$.

We have

$$
\sup _{a \in \mathbb{R}^{d}} \frac{\nu(B(a, R))}{R^{\alpha}} \leq \sup _{a} \frac{\nu\left(B\left(a, \frac{\delta}{\epsilon_{n+1}}\right)\right)}{\left(\frac{\delta}{\epsilon_{n}}\right)^{\alpha}}=\sup _{a} \frac{\nu\left(B\left(a, \frac{\delta}{\epsilon_{n+1}}\right)\right)}{\left(\frac{\delta}{\epsilon_{n+1}}\right)^{\alpha}} \cdot \frac{\epsilon_{n}^{\alpha}}{\epsilon_{n+1}^{\alpha}} \leq \frac{1}{\delta^{\alpha}} \frac{B}{C} M^{\alpha} \text {. }
$$

This shows that $\mathcal{D}_{\alpha}(\nu)<\infty$, so $\operatorname{dim} \nu \leq \alpha$.

Definition 3.9. Let $\nu$ be a locally finite Borel measure on $\mathbb{R}^{d}$. The $d$-lower Beurling density (or simply the lower Beurling density) of $\nu$ is defined by

$$
\mathcal{D}^{-}(\nu)=\liminf _{R \rightarrow \infty} \inf _{x \in \mathbb{R}^{d}} \frac{\nu(x+R Q)}{R^{d}} .
$$

Theorem 3.10. Let $\mu$ be a compactly supported Borel probability measure on $\mathbb{R}^{d}$. Suppose $\mu$ has a Bessel measure $\nu$ of positive lower Beurling density. Then $\mu$ is absolutely continuous with respect to the Lebesgue measure, and its Radon-Nikodym derivative is in $L^{2}\left(\mathbb{R}^{d}\right)$. 
Proof. Since $\nu$ has positive lower Beurling density, there exist $R>0$ and $\delta>0$ such that

$$
\frac{\nu(x+R Q)}{R^{d}} \geq \delta \text { for all } x \in \mathbb{R}^{d}
$$

Then

$$
\nu(x+R Q) \geq \delta R^{d}=: C>0, \text { for all } x \in \mathbb{R}^{d} .
$$

With Theorem 2.11, the measure $\nu^{\prime}=\sum_{k \in \mathbb{Z}^{d}} \nu(R(k+Q)) \delta_{R k}$ is also a Bessel measure for $\mu$. Note that $\nu(R(k+Q)) \geq C$ for all $k \in \mathbb{Z}$. Using Proposition 2.3. the convolution of $\nu^{\prime}$ with the probability measure $\frac{1}{R^{d}} \chi_{R Q}(x) d x$, we obtain that the measure

$$
\nu^{\prime \prime}=\frac{1}{R^{d}} \sum_{k \in \mathbb{Z}^{d}} \nu(R(k+Q)) \chi_{R k+R Q}(x) d x=: H(x) d x
$$

is a Bessel measure for $\mu$. But the previous remarks show that $H(x) \geq \frac{1}{R^{d}} C$ for all $x \in \mathbb{R}^{d}$. Therefore we have

$$
\int|\widehat{\mu}(x)|^{2} d x \leq \frac{R^{d}}{C} \int|\widehat{\mu}(x)|^{2} H(x) d x \leq \frac{R^{d}}{C} B,
$$

where $B$ is the Bessel bound for the Bessel measure $H(x) d x$.

This means that $\hat{\mu}$ is in $L^{2}\left(\mathbb{R}^{d}\right)$. Then there exists some function $g \in L^{2}\left(\mathbb{R}^{d}\right)$ whose Fourier transform $\hat{g}$ is $\hat{\mu}$.

Take $f$ to be an arbitrary $C^{\infty}$, compactly supported function on $\mathbb{R}^{d}$. Then $\hat{f}$ is in $L^{1}\left(\mathbb{R}^{d}\right) \cap L^{2}\left(\mathbb{R}^{d}\right)$, and using the Parseval relation (see e.g., Kat04, Chapter VI.2]), we have

$$
\int f d \mu=\int \widehat{f}(\xi) \widehat{\mu}(-\xi) d \xi=\int \widehat{f}(\xi) \overline{\widehat{\mu}(\xi)} d \xi=\int \widehat{f}(\xi) \overline{\hat{g}(\xi)} d \xi=\int f(x) \overline{g(x)} d x .
$$

Since $f$ is arbitrary, it follows that $d \mu=\bar{g} d x$. Note that in particular this implies that $g$ is non-negative.

For Lebesgue measure restricted to the unit interval, a necessary condition for a frame measure is that the Beurling dimension is 1 . We will prove this using a result in OCS02 concerning equivalent norms for the Paley-Wiener space. Recall that the Paley-Wiener space $P W_{\pi}$ is the collection of entire functions which are of exponential type $\pi$ and are square integrable on the real axis. By the Paley-Wiener theorem [PW87], this consists of all functions $f$ such that $f(z)=\int_{-\pi}^{\pi} g(t) e^{i t z} d t$ for some $g \in L^{2}([-\pi, \pi])$. By reparametrizing, we consider $f(z)=\int_{-1 / 2}^{1 / 2} g(t) e^{-2 \pi i t z} d t$ for some $g \in L^{2}([-1 / 2,1 / 2])$. Landau's inequality Lan67 implies that if $\left\{\lambda_{k}\right\}$ is a sequence such that there exist constants $0<A, B<\infty$ such that for every $f \in P W_{\pi}$,

$$
A \int_{\mathbb{R}}|f(t)|^{2} d t \leq \sum_{k \in \mathbb{Z}}\left|f\left(\lambda_{k}\right)\right|^{2} \leq B \int_{\mathbb{R}}|f(t)|^{2} d t
$$

then the Beurling density of $\left\{\lambda_{k}\right\}$ satisfies the inequalities

$$
1 \leq \mathcal{D}^{-}\left(\left\{\lambda_{k}\right\}\right) \leq \mathcal{D}^{+}\left(\left\{\lambda_{k}\right\}\right)<\infty .
$$


If (3.9) is satisfied, then $\left\{\lambda_{k}\right\}$ is a sampling set for $P W_{\pi}$, and is equivalent to $\left\{e_{\lambda_{k}}\right\} \subset L^{2}([-1 / 2,1 / 2])$ being a Fourier frame. From page 798 of OCS02]: for $r, \delta>0$ and a measure $\nu$, we define the sequence

$$
\Lambda_{\nu}(r, \delta)=\{k r: k \in \mathbb{Z}, \nu([k r,(k+1) r)) \geq \delta\} .
$$

Proposition 3.11. A Borel measure $\nu$ yields an equivalent norm for $P W_{\pi}$, i.e., the measure $\nu$ is a frame measure for the Lebesgue measure on $[-1 / 2,1 / 2]$, if and only if the following hold:

(i) There exists a positive constant $C$ such that $\nu([x, x+1)) \leq C$ for all $x \in \mathbb{R}$.

(ii) For all sufficiently small $r>0$ there exists a $\delta=\delta(r)>0$ such that $\Lambda_{\nu}(r, \delta)$ is a sampling set for $P W_{\pi}$.

Theorem 3.12. If $\mu=\left.\lambda\right|_{\left[-\frac{1}{2}, \frac{1}{2}\right]}$, and if $\nu$ is a frame measure for $\mu$, then $\mathcal{D}^{-}(\nu)>0$, and hence $\operatorname{dim}(\nu)=1$.

Proof. The measure $\nu$ is a frame measure for $\mu$ if and only if for every $f$ in the Paley-Wiener space $P W_{\pi}$ we have

$$
A \int|f(t)|^{2} d \lambda(t) \leq \int|f(t)|^{2} d \nu(t) \leq B \int|f(t)|^{2} d \lambda(t)
$$

and thus $\nu$ yields an equivalent norm for $P W_{\pi}$. By the preceding proposition we have that there exist an $r>0$ and a $\delta>0$ such that the sequence $\Lambda_{\nu}(\delta, r)$ is a sampling sequence for $P W$.

For $x, R \in \mathbb{R}$, we define the set

$$
W_{r}(x, R)=\{k r: k \in \mathbb{Z} ; \nu([k r,(k+1) r)) \geq \delta ;[k r,(k+1) r) \subset x+R Q\} .
$$

Note that $W_{r}(x, R) \subset \Lambda_{\nu}(\delta, r) \cap(x+R Q)$ and moreover the latter set is larger than the former by at most 1 element. Thus, we have for every $x \in \mathbb{R}$ and $R>0$,

$$
\begin{aligned}
\nu(x+R Q) & \geq \sum_{k r \in W_{r}(x, R)} \nu([k r,(k+1) r)) \geq \delta \cdot \# W_{r}(x, R) \\
& \geq \delta \cdot\left(\#\left(\Lambda_{\nu}(\delta, r) \cap(x+R Q)\right)-1\right) .
\end{aligned}
$$

Therefore, we have that

$$
\begin{aligned}
\mathcal{D}^{-}(\nu) & =\liminf _{R \rightarrow \infty} \inf _{x \in \mathbb{R}} \frac{\nu(x+R Q)}{R} \\
& \geq \delta \liminf _{R \rightarrow \infty} \inf _{x \in \mathbb{R}} \frac{\#\left(\Lambda_{\nu}(\delta, r) \cap(x+R Q)\right)-1}{R} \\
& =\delta \mathcal{D}^{-}\left(\left(\Lambda_{\nu}(\delta, r)\right)\right. \\
& >0
\end{aligned}
$$

by (3.10). We have that $\mathcal{D}^{-}(\nu) \leq \mathcal{D}_{1}(\nu)<\infty$ by Theorem 3.6.

\section{Affine iterated FunCtion systems}

Our motivating examples, measures restricted to Cantor type sets, are invariant measures for iterated function systems. For all such measures, we obtain an upper bound on the Beurling dimension of any Bessel measure. For some such measures, as demonstrated in Theorems 4.6 and 4.8, the structure of the iterated function system provides a way of constructing frame measures. Specifically, the measure $\mu_{4}$ of [JP98] is one such case; unfortunately, the measure $\mu_{3}$ for the usual Cantor set is not one of those cases. 
Definition 4.1. Let $R$ be a $d \times d$ expanding integer matrix, i.e., all eigenvalues $\lambda$ satisfy $|\lambda|>1$. Let $B$ be a finite subset of $\mathbb{Z}^{d}$ of cardinality $\# B=: N$. We consider the following affine iterated function system:

$$
\tau_{b}(x)=R^{-1}(x+b) \quad\left(x \in \mathbb{R}^{d}, b \in B\right) .
$$

Since $R$ is expanding, the maps $\tau_{b}$ are contractions (in an appropriate metric equivalent to the Euclidean one), and therefore Hutchinson's theorem can be applied Hut81]: there exists a unique compact set $X=X_{B} \subset \mathbb{R}^{d}$ such that

$$
X=\bigcup_{b \in B} \tau_{b}(X) .
$$

The set $X_{B}$ is called the attractor of the affine IFS. Moreover,

$$
X_{B}=\left\{\sum_{k=1}^{\infty} R^{-k} b_{k}: b_{k} \in B \text { for all } k \in \mathbb{N}\right\} \text {. }
$$

There exists a unique Borel probability measure $\mu=\mu_{B}$ on $\mathbb{R}^{d}$ such that

$$
\int f d \mu=\frac{1}{N} \sum_{b \in B} \int f \circ \tau_{b} d \mu
$$

for all compactly supported continuous functions $f$ on $\mathbb{R}^{d}$. Moreover, the measure $\mu_{B}$ is supported on the set $X_{B}$. The measure $\mu_{B}$ is called the invariant measure of the affine IFS.

We say that the affine IFS has no overlap if $\mu_{B}\left(\tau_{b}\left(X_{B}\right) \cap \tau_{b^{\prime}}\left(X_{B}\right)\right)=0$ for all $b \neq b^{\prime}$ in $B$.

Lemma 4.2. If the IFS has no overlap, and $E$ is a Borel set, then

$$
\mu_{B}\left(\tau_{b}(E)\right)=\frac{1}{N} \mu_{B}(E) .
$$

In particular,

$$
\mu_{B}\left(\tau_{b_{1}} \ldots \tau_{b_{n}}\left(X_{B}\right)\right)=\frac{1}{N^{n}} \quad\left(n \in \mathbb{N}, b_{1}, \ldots, b_{n} \in B\right) .
$$

Proof. From the invariance equation we have that

$$
\mu_{B}(E)=\frac{1}{N} \sum_{b \in B} \mu_{B}\left(\tau_{b}^{-1}(E)\right)
$$

so if $\mu_{B}(E)=0$, then $\mu_{B}\left(\tau_{b}^{-1}(E)\right)=0$ for all $b \in B$. Also,

$$
\mu_{B}\left(\tau_{b}(E)\right)=\frac{1}{N} \sum_{b^{\prime} \in B} \mu_{B}\left(\tau_{b^{\prime}}^{-1}\left(\tau_{b}(E)\right)\right)=\frac{1}{N} \mu_{B}(E)+\frac{1}{N} \sum_{b^{\prime} \neq b} \mu_{B}\left(\tau_{b^{\prime}}^{-1}\left(\tau_{b}(E)\right)\right) .
$$

But using the no overlap and the fact that $\mu_{B}$ is supported on $X_{B}$, we have

$$
\begin{aligned}
\mu_{B}\left(\tau_{b^{\prime}}\left(\tau_{b}(E)\right)\right) & \leq \mu_{B}\left(\tau_{b^{\prime}}^{-1}\left(\tau_{b}\left(X_{B}\right)\right)\right)=\mu_{B}\left(X_{B} \cap \tau_{b^{\prime}}^{-1}\left(\tau_{b}\left(X_{B}\right)\right)\right) \\
& \leq=\mu_{B}\left(\tau_{b^{\prime}}^{-1}\left(\tau_{b^{\prime}}\left(X_{B}\right) \cap \tau_{b}\left(X_{B}\right)\right)\right)=0 .
\end{aligned}
$$

This proves 4.5.).

Applying (4.5), we obtain by induction that

$$
\mu_{B}\left(\tau_{b_{1}} \ldots \tau_{b_{n}}\left(X_{B}\right)\right)=\frac{1}{N^{n}}
$$

for all $n \in \mathbb{N}$ and $b_{1}, \ldots, b_{n} \in B$. 
Theorem 4.3. In dimenson $d=1$, let $(R, B)$ be an affine IFS with no overlap, $N=\# B$, with $\mu_{B}$ the associated invariant measure. If $\nu$ is a Bessel measure for $\mu_{B}$, the Beurling dimension of $\nu$ satisfies

$$
\operatorname{dim} \nu \leq \frac{\log N}{\log R}
$$

Proof. We prove that $\mu_{B}$ is occasionally $\frac{\log N}{\log R}$-dimensional, whence the statement follows from Theorem 3.8. Let $b_{0} \in B$ and let $E_{n}=\tau_{b_{0}}^{n}\left(X_{B}\right) ; \operatorname{since} \operatorname{diam}\left(E_{n}\right)=$ $R^{-n} \operatorname{diam}\left(E_{0}\right)$, we have that $\operatorname{diam}\left(E_{n}\right) \rightarrow 0$ and $\sup \frac{\operatorname{diam}\left(E_{n}\right)}{\operatorname{diam}\left(E_{n+1}\right)}=R$. Moreover, by (4.6), $\mu_{B}\left(E_{n}\right)=\frac{1}{N^{n}}$. We have

$$
\begin{aligned}
\operatorname{diam}\left(E_{n}\right)^{\log N / \log R} & =\left(R^{-n}\right)^{\log N / \log R} \operatorname{diam}\left(E_{0}\right)^{\log N / \log R} \\
& =N^{-n} \operatorname{diam}\left(E_{0}\right)^{\log N / \log R},
\end{aligned}
$$

and therefore (3.7) is satisfied with $c_{1}=c_{2}=\operatorname{diam}\left(E_{0}\right)^{\log N / \log R}$.

Proposition 4.4. Let $(R, B)$ be an affine IFS with no overlap, $N=\# B$. On the compact space $B^{\mathbb{N}}$ consider the product probability measure where each digit in $B$ has probability $1 / N$. Define the encoding $\operatorname{map} \mathcal{E}_{B}: B^{\mathbb{N}} \rightarrow X_{B}$,

$$
\mathcal{E}_{B}\left(b_{1} b_{2} \ldots\right)=R^{-1} b_{1}+R^{-2} b_{2}+\ldots
$$

Then $\mathcal{E}_{B}$ is onto, measure preserving and one-to-one on a set of full measure.

Proof. Equation (4.6) proves that $\mathcal{E}_{B}$ is measure preserving. The fact that $\mathcal{E}_{B}$ is onto follows from (4.3).

We now check that $\mathcal{E}_{B}$ is one-to-one on a set of full measure. Let

$$
E:=\bigcup_{b \neq b^{\prime}}\left(\tau_{b}\left(X_{B}\right) \cap \tau_{b^{\prime}}\left(X_{B}\right)\right), \quad F:=E \cup \bigcup_{n \geq 1, b_{1}, \ldots, b_{n} \in B} \tau_{b_{1}} \ldots \tau_{b_{n}}(E) .
$$

From the computations above, we see that $F$ has measure zero. It is also clear that $\tau_{b}^{-1}(F) \supset F$ for all $b \in B$.

Take $x \in X_{B} \backslash F$. Suppose $x=R^{-1} b_{1}+R^{-2} b_{2}+\ldots$ (by (4.3)). Then $x=\tau_{b_{1}}(y)$ with $y=R^{-1} b_{2}+R^{-2} b_{3}+\ldots$ Since $x \notin F \supset E, b_{1}$ is uniquely determined by $x$. We have $y=\tau_{b_{1}}^{-1}(x) \notin \tau_{b_{1}}^{-1}(F)$, so $y \notin F$. Repeating the argument, $b_{2}$ is uniquely determined, and so on for all $b_{n}$. Therefore $\mathcal{E}_{B}$ is one-to-one on the set of full measure $\mathcal{E}_{B}^{-1}\left(X_{B} \backslash F\right)$.

Definition 4.5. For two subsets $A, B$ of $\mathbb{R}^{d}$ we say that $A \oplus B=C$ if for every element $c \in C$ there exist unique $a \in A$ and $b \in B$ such that $a+b=c$.

Theorem 4.6. Let $(R, B)$ be an affine IFS with no overlap. Suppose there exists a finite set $C$ such that $B \oplus C=: D$ is a complete set of representatives for $\mathbb{Z}^{d} / R \mathbb{Z}^{d}$ and the affine IFS $(R, C)$ has no overlap. Then $\mu_{B}$ has a Plancherel measure supported on a lattice. More precisely, the measure $\mu_{D}$ has as its spectrum a lattice $\Gamma$, and the measure

$$
\nu=\sum_{\gamma \in \Gamma}\left|\widehat{\mu_{C}}(\gamma)\right|^{2} \delta_{\gamma}
$$

is a Plancherel measure for $\mu_{B}$. More generally, if $\nu$ is a Bessel/frame measure for $\mu_{D}$, then $\left|\widehat{\mu}_{C}\right|^{2} d \nu$ is a Bessel/frame measure for $\mu_{B}$ with the same bounds. 
Proof. The fact that $\mu_{D}$ is the Lebesgue measure on a set that tiles $\mathbb{R}^{d}$ by some lattice $\Gamma$ and that there is no overlap for $\mu_{D}$ are contained in [CHR97. We will use Proposition 2.9.

Define the maps $\Phi: D^{\mathbb{N}} \rightarrow B^{\mathbb{N}} \times C^{\mathbb{N}}$ and $+: X_{B} \times X_{C} \rightarrow X_{D}$ :

$$
\begin{gathered}
\Phi\left(d_{1} d_{2} \ldots\right)=\left(b_{1} b_{2} \ldots, c_{1} c_{2} \ldots\right), \text { where } d_{1}=b_{1}+c_{1}, d_{2}=b_{2}+c_{2}, \ldots, \\
+(x, y)=x+y .
\end{gathered}
$$

The diagram below is commutative:

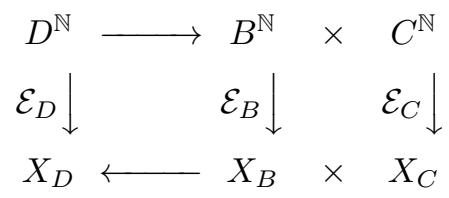

It is easy to check that $\Phi$ is bijective and measure preserving. From Proposition 4.4 we conclude that the map + is bijective on sets of full measure and is measure preserving. Since + is measure preserving, we see that $\mu_{B} * \mu_{C}=\mu_{D}$ (alternatively, consider the Fourier transforms).

We can define the map $p: X_{D} \rightarrow X_{B}$,

$$
p(x+y)=x \quad\left(x \in X_{B}, y \in X_{C}\right) .
$$

We will prove that for $f \in L^{1}\left(\mu_{B}\right)$ the function (see Proposition 2.7 for the definition) $P f=P_{\mu_{B}, \mu_{C}} f=f \circ p$. Indeed, for a bounded Borel function $g$ we have

$$
\begin{aligned}
\int g d\left(\left(f d \mu_{B}\right) * \mu_{C}\right) & =\iint g(x+y) f(x) d \mu_{B}(x) d \mu_{C}(y) \\
= & \iint g(x+y) f \circ p(x+y) d \mu_{B}(x) d \mu_{C}(y)=\int g f \circ p d\left(\mu_{B} * \mu_{C}\right) .
\end{aligned}
$$

In addition, we have

$$
\begin{aligned}
\int f \circ p d\left(\mu_{B} * \mu_{C}\right) & =\iint f(p(x+y)) d \mu_{B}(x) d \mu_{C}(y)=\iint f(x) d \mu_{B}(x) d \mu_{C}(y) \\
& =\int f d \mu_{B} .
\end{aligned}
$$

Hence

$$
\|P f\|_{L^{2}\left(\mu_{B} * \mu_{C}\right)}=\|f\|_{L^{2}\left(\mu_{B}\right)} \quad\left(f \in L^{2}\left(\mu_{B}\right)\right) .
$$

Everything follows from Proposition 2.9 .

Corollary 4.7. Let $\mu_{4}$ be the invariant measure for the affine IFS with $R=4$ and $B=\{0,2\}$, and let $\mu_{4}^{\prime}$ be the invariant measure for the affine IFS with $R=4$ and $C=\{0,1\}$. Then $\left|\widehat{\mu_{4}^{\prime}}(x)\right|^{2} d x$ and $\sum_{n \in \mathbb{Z}}\left|\widehat{\mu_{4}^{\prime}}(n)\right|^{2} \delta_{n}$ are Plancherel measures for $\mu_{4}$. Therefore, $\left\{\left|\widehat{\mu_{4}^{\prime}}(n)\right| e_{n}\right\} \subset L^{2}\left(\mu_{4}\right)$ is a Parseval frame.

Moreover, if $\left\{e_{\lambda_{n}}\right\} \subset L^{2}[0,1]$ is a Fourier frame, then $\left\{\left|\widehat{\mu_{4}^{\prime}}\left(\lambda_{n}\right)\right| e_{\lambda_{n}}\right\} \subset L^{2}\left(\mu_{4}\right)$ is a frame, with the same frame bounds.

Proof. With $B=\{0,2\}$ and $C=\{0,1\}$ we have $B \oplus C=\{0,1,2,3\}=D$, and the measures $\mu_{B}, \mu_{C}$ have no overlap. The measure $\mu_{D}$ is the Lebesgue measure on $[0,1]$. Then everything follows from Theorem 4.6 
In DHSW11 it is shown that a Bessel sequence of exponentials for $\mu_{4}$ must have Beurling dimension at most $\frac{1}{2}$. We point out here that the sequence

$$
\mathcal{Y}:=\left\{n:\left|\widehat{\mu_{4}^{\prime}}(n)\right|^{2} \neq 0\right\}
$$

has Beurling dimension 1. Indeed, we can determine the zero set of $\widehat{\mu_{4}^{\prime}}(t)$ as is done in JP98]:

$$
\widehat{\mu_{4}^{\prime}}(t)=\prod_{n=0}^{\infty} \frac{1}{2}\left(1+e^{2 \pi i t / 4^{n}}\right)=e^{2 \pi i t / 3} \cdot \prod_{n=1}^{\infty} \cos \left(\frac{\pi t}{4^{n}}\right) .
$$

From this we see that the zero set is

$$
\mathcal{Z}\left(\widehat{\mu_{4}^{\prime}}(t)\right)=\left\{4^{n}(4 \mathbb{Z}+2): n=0,1, \ldots\right\}
$$

which has Beurling density $\mathcal{D}_{1}\left(\mathcal{Z}\left(\widehat{\mu_{4}^{\prime}}(t)\right)\right)=\frac{1}{3}$. Therefore, the sequence of exponentials

$$
\left\{\left|\widehat{\mu_{4}^{\prime}}(n)\right| e_{n}: n \in \mathcal{Y}\right\}
$$

which forms a Parseval frame in $L^{2}\left(\widehat{\mu}_{4}\right)$ by Corollary 4.7, has Beurling density $\frac{2}{3}$, and hence Beurling dimension 1.

However, when we consider this sequence as a measure $\nu=\sum_{n \in \mathbb{Z}}\left|\widehat{\mu_{4}^{\prime}}(n)\right|^{2} \delta_{n}$, we must have by Theorem 3.8 that $\nu$ has Beurling dimension at most $\frac{1}{2}$.

Theorem 4.8. Suppose that B, $C, D$ are as in Theorem 4.6. For functions $f \in$ $L^{2}\left(\mu_{B}\right)$ such that $P f:=P_{\mu_{B}, \mu_{C}} f$ is continuous on the support of $\mu_{D}$ and its Fourier transform is in $L^{1}\left(\mathbb{R}^{d}\right)$, we have the following Fourier reconstruction theorem: for every $t$ in the support of $\mu_{B}$,

$$
f(t)=\int\left(\widehat{f d \mu_{B}}(x) \cdot \widehat{\mu_{C}}(x)\right) e^{2 \pi i t \cdot x} d x .
$$

Proof. Since $\mu_{D}$ is a Lebesgue measure restricted to a set, we have by the Fourier inversion theorem that for $t$ in the support of $\mu_{D}$,

$$
\begin{aligned}
P f(t) & =\int \widehat{P f}(x) e^{2 \pi i x \cdot t} d x \\
& =\int\left\{\int P f(y) e^{-2 \pi i y \cdot x} d \mu_{D}(y)\right\} e^{2 \pi i x \cdot t} d x \\
& =\int\left\{\int e^{-2 \pi i y \cdot x} \operatorname{Pf}(y) d\left(\mu_{B} * \mu_{C}\right)(y)\right\} e^{2 \pi i x \cdot t} d x \\
& =\int\left\{\int e^{-2 \pi i y \cdot x} d\left(\left(f d \mu_{B}\right) * \mu_{C}\right)(y)\right\} e^{2 \pi i x \cdot t} d x \\
& =\int\left(\left(f d \mu_{B}\right) * \mu_{C}\right)^{\wedge}(x) e^{2 \pi i x \cdot t} d x \\
& =\int\left(\widehat{f d \mu_{B}}(x) \cdot \widehat{\mu_{C}}(x)\right) e^{2 \pi i x \cdot t} d x .
\end{aligned}
$$

However, for $t$ in the support of $\mu_{B}, P f(t)=f(t)$ as in the proof of Theorem 4.6. 


\section{REFERENCES}

[CHR97] J.-P. Conze, L. Hervé, and A. Raugi. Pavages auto-affines, opérateurs de transfert et critères de réseau dans $\mathbf{R}^{d}$. Bol. Soc. Brasil. Mat. (N.S.), 28(1):1-42, 1997. MR.1444447(99a:52034)

[CKS08] Wojciech Czaja, Gitta Kutyniok, and Darrin Speegle. Beurling dimension of Gabor pseudoframes for affine subspaces. J. Fourier Anal. Appl., 14(4):514-537, 2008. MR2421575 (2009k:42062)

[DHS09] Dorin Ervin Dutkay, Deguang Han, and Qiyu Sun. On the spectra of a Cantor measure. Adv. Math., 221(1):251-276, 2009. MR2509326 (2010f:28013)

[DHSW11] Dorin Ervin Dutkay, Deguang Han, Qiyu Sun, and Eric Weber. On the Beurling dimension of exponential frames. Adv. Math., 226:285-297, 2011. MR2735759 (2012a:42058)

[DHW11] Dorin Ervin Dutkay, Deguang Han, and Eric Weber. Bessel sequences of exponentials on fractal measures. J. Functional Anal., to appear, 2011. MR 2826404

[DS52] R. Duffin and A. Schaeffer. A class of nonharmonic Fourier series. Trans. Amer. Math. Soc., 72:341-366, 1952. MR0047179(13:839a)

[GH03] J.-P. Gabardo and D. Han. Frames associated with measurable spaces. Adv. Comput. Math., 18(2-4):127-147, 2003. Frames. MR.1968116 (2004b:42062)

[HL08] Tian-You Hu and Ka-Sing Lau. Spectral property of the Bernoulli convolutions. Adv. Math., 219(2):554-567, 2008. MR2435649 (2010a:42094)

[Hut81] John E. Hutchinson. Fractals and self-similarity. Indiana Univ. Math. J., 30(5):713747, 1981. MR625600 (82h:49026)

[IP00] Alex Iosevich and Steen Pedersen. How large are the spectral gaps? Pacific J. Math., 192(2):307-314, 2000. MR1744572 (2001b:42038)

[JKS07] Palle E. T. Jorgensen, Keri A. Kornelson, and Karen L. Shuman. Affine systems: asymptotics at infinity for fractal measures. Acta Appl. Math., 98(3):181-222, 2007. MR2338387 (2008i:42013)

[JP98] Palle E. T. Jorgensen and Steen Pedersen. Dense analytic subspaces in fractal $L^{2}$ spaces. J. Anal. Math., 75:185-228, 1998. MR1655831 (2000a:46045)

[JP99] Palle E. T. Jorgensen and Steen Pedersen. Spectral pairs in Cartesian coordinates. J. Fourier Anal. Appl., 5(4):285-302, 1999. MR1700084 (2002d:42027)

[Kat04] Yitzhak Katznelson. An introduction to harmonic analysis. Cambridge Mathematical Library. Cambridge University Press, Cambridge, third edition, 2004. MR 2039503 (2005d:43001)

[Lan67] H. J. Landau. Necessary density conditions for sampling and interpolation of certain entire functions. Acta Math., 117:37-52, 1967. MR.0222554 (36:5604)

[Li07] Jian-Lin Li. $\mu_{M, D}$-orthogonality and compatible pair. J. Funct. Anal., 244(2):628-638, 2007. MR,2297038 (2008h:42046)

[LS02] Yurii I. Lyubarskii and Kristian Seip. Weighted Paley-Wiener spaces. J. Amer. Math. Soc., 15(4):979-1006 (electronic), 2002. MR1915824(2003m:46039)

[ŁW06] Izabella Łaba and Yang Wang. Some properties of spectral measures. Appl. Comput. Harmon. Anal., 20(1):149-157, 2006. MR2200934 (2007e:28001)

[MZ09] U. Molter and L. Zuberman. A fractal Plancherel theorem. Real Anal. Exchange, 34(1):69-85, 2009. MR2527123 (2010e:42013)

[OCS02] Joaquim Ortega-Cerdà and Kristian Seip. Fourier frames. Ann. of Math. (2), 155(3):789-806, 2002. MR.1923965 (2003k:42055)

[PW87] Raymond E. A. C. Paley and Norbert Wiener. Fourier transforms in the complex domain. Reprint of the 1934 original. American Mathematical Society Colloquium Publications, 19. American Mathematical Society, Providence, RI, $1987 \mathrm{x}+184 \mathrm{pp}$. MR.1451142 (98a:01023)

[Str90] Robert S. Strichartz. Self-similar measures and their Fourier transforms. I. Indiana Univ. Math. J., 39(3):797-817, 1990. MR1078738 (92k:42015)

[Str93a] Robert S. Strichartz. Self-similar measures and their Fourier transforms. II. Trans. Amer. Math. Soc., 336(1):335-361, 1993. MR1081941(93e:42023)

[Str93b] Robert S. Strichartz. Self-similar measures and their Fourier transforms. III. Indiana Univ. Math. J., 42(2):367-411, 1993. MR.1237052 (94j:42025)

[Str00] Robert S. Strichartz. Mock Fourier series and transforms associated with certain Cantor measures. J. Anal. Math., 81:209-238, 2000. MR1785282(2001i:42009) 
[Str06] Robert S. Strichartz. Convergence of mock Fourier series. J. Anal. Math., 99:333-353, 2006. MR2279556 (2007j:42004)

[Yua08] Yan-Bo Yuan. Analysis of $\mu_{R, D}$-orthogonality in affine iterated function systems. Acta Appl. Math., 104(2):151-159, 2008. MR2443273(2010h:28025)

Department of Mathematics, University of Central Florida, 4000 Central Florida Boulevard, P.O. Box 161364, Orlando, Florida 32816-1364

E-mail address: Dorin.Dutkay@ucf.edu

Department of Mathematics, University of Central Florida, 4000 Central Florida Boulevard, P.O. Box 161364, Orlando, Florida 32816-1364

E-mail address: deguang.han@ucf.edu

Department of Mathematics, 396 Carver Hall, Iowa State University, Ames, Iowa 50011

E-mail address: esweber@iastate.edu 